\title{
FIRST NONLINEAR SYZYGIES OF IDEALS ASSOCIATED TO GRAPHS
}

\author{
OSCAR FERNÁNDEZ-RAMOS AND PHILIPPE GIMENEZ
}

\begin{abstract}
Consider an ideal $I \subset K\left[x_{1}, \ldots, x_{n}\right]$, with $K$ an arbitrary field, generated by monomials of degree two. Assuming that $I$ does not have a linear resolution, we determine the step $s$ of the minimal graded free resolution of $I$ where nonlinear syzygies first appear, we show that at this step of the resolution nonlinear syzygies are concentrated in degree $s+3$, and we compute the corresponding graded Betti number $\beta_{s, s+3}$. The multidegrees of these nonlinear syzygies are also determined and the corresponding multigraded Betti numbers are shown to be all equal to 1 .
\end{abstract}

\section{INTRODUCTION}

Let $R:=K\left[x_{1}, \ldots, x_{n}\right]$ be the polynomial ring in $n$ variables over an arbitrary field $K$, and let $I$ be an ideal in $R$ generated by a finite set $\mathbf{f}=\left\{f_{1}, \ldots, f_{m}\right\}$ of distinct monomials in $R$ of degree two. Associated to $I$ there is a graph $G(I)$ with vertex and edge sets $V_{G(I)}:=\{(1), \ldots,(n)\}$ and $E_{G(I)}:=\left\{(i, j) ; 1 \leq i \leq j \leq n / x_{i} x_{j} \in I\right\}$, respectively, and we shall say that $I$ is an ideal associated to a graph. There is another graph related to the ideal $I$ that will be featured in this paper, namely, the (simple) complement $G(I)^{c}$ of $G(I)$ with vertex set $V_{G(I)}$ and edge set $E_{G(I)^{c}}:=\left\{(i, j) ; 1 \leq i<j \leq n /(i, j) \notin E_{G(I)}\right\}$. Note that the graph $G(I)$ may have loops while $G(I)^{c}$ is always a simple graph. When $I$ is squarefree, i.e., when $G(I)$ is simple, $I$ is called an edge ideal. In this connection we mention the survey in Villarreal's book [13, Chapter 6].

As a general aim, one would like to establish a correspondence between algebraic properties of the ideal $I$ (or the ring $R / I$, or the subalgebra $K[\mathbf{f}] \subset R$ ) and the graph theoretical data of $G(I)$ (or of any other graph associated to $I$ such as $\left.G(I)^{c}\right)$. Two illustrations of this correspondence are the combinatorial characterizations of normality and polarizability of the subalgebra $K[\mathbf{f}] \subset R$ given in [12] and [2], respectively. In this note, we shall focus on properties of $I$ related to its minimal graded free resolution

$$
0 \rightarrow \bigoplus_{j} R(-j)^{\beta_{p, j}} \longrightarrow \cdots \longrightarrow \bigoplus_{j} R(-j)^{\beta_{0, j}} \longrightarrow I \rightarrow 0
$$

where $R(-j)$ denotes the graded $R$-module obtained by shifting the degrees of homogeneous elements in $R$ by $j$. The number of generators of degree $j$ in the $i$ th syzygy module is denoted by $\beta_{i, j}$. These numbers do not depend on the minimal graded free resolution and are called the graded Betti numbers of $I$. The numerical information contained in the minimal graded free resolution of $I$ (degrees of the syzygies and graded Betti numbers) can be displayed on a table with $p+1$ columns labeled $0,1, \ldots, p$ that correspond to the steps in the resolution and with rows corresponding to degrees, where the entry in the $j$ th row of the $i$ th column is $\beta_{i, i+j}$. This table is called the Betti diagram of $I$; see [1] and [3, page 7]. The size of the Betti diagram of $I$ is a measure of the size of the minimal graded free resolution of $I$ and hence, in some sense, a measure of the complexity of the ideal $I$. By [3, Proposition 1.9], the first row (with a nonzero entry) of the Betti diagram is the one labeled by the initial 
degree $\delta$ of $I$, i.e., the smallest integer $j$ such that $\beta_{0, j} \neq 0$. In our situation, $\delta=2$ and in fact $\beta_{0, j}=0$ for all $j \neq 2$. The last row (with a nonzero entry) of the diagram is the one labeled by $m$, the Castelnuovo-Mumford regularity of $I$. The number of rows of the Betti diagram is thus $m-\delta+1$, and the number of columns is related to the depth of $R / I$ by the Auslander-Buchsbaum formula, $p+1=n-\operatorname{depth}(R / I)$.

We say that $I$ has a linear resolution if $\beta_{i, j}=0$ whenever $j \neq i+2$. In terms of the Betti diagram, $I$ has a linear resolution if and only if the diagram has only one row. The classical result of Fröberg [ 6 , Theorem 1] characterizes edge ideals with linear resolution. It has recently been recovered by Eisenbud, Green, Hulek and Popescu that provide some additional information. When the resolution of $I$ is nonlinear, they show in [4, Theorem 2.1] that the step of the minimal graded free resolution of $I$ where nonlinear syzygies first appear is determined by the length of the shortest cycle in $G(I)^{c}$ having no chord. A similar result for ideals associated to graphs that are not squarefree can be deduced from [4, Proposition 2.3]. In this note, we prove the following more precise result:

Theorem 1.1. Consider an ideal $I \subset K\left[x_{1}, \ldots, x_{n}\right]$ generated by monomials of degree two, with $K$ an arbitrary field. Let $G(I)$ be the graph associated to $I$ and let $G(I)^{c}$ be its complement. For all $d \geq 3$, set $i_{d}:=\min _{1 \leq i \leq p}\left\{i ; \beta_{i, i+d} \neq 0\right\}$ if $\beta_{i, i+d} \neq 0$ for some $i \geq 1$, $i_{d}:=0$ otherwise. Then:

(1) $i_{3}=1$ if and only if $G(I)$ has at least one induced subgraph consisting of two disjoint edges. When this occurs, $\beta_{1,4}$ is the number of such induced subgraphs of $G(I)$, and $\beta_{1, j}=0$ for all $j>4$.

(2) $i_{3}>1$ if and only if $G(I)$ has no induced subgraph consisting of two disjoint edges and $G(I)^{c}$ has at least one induced cycle of length $\geq 5$. When this occurs, $i_{3}=r-3$ where $r$ is the smallest integer $\geq 5$ such that $G(I)^{c}$ has an induced $r$-cycle, $\beta_{i_{3}, i_{3}+3}$ is the number of induced $r$-cycles in $G(I)^{c}$, and $\beta_{i, j}=0$ for all $i \leq i_{3}$ and $j>i_{3}+3$.

(3) $i_{3}=0$ if and only if $G(I)$ has no induced subgraph consisting of two disjoint edges and $G(I)^{c}$ is chordal. When this occurs, I has a linear resolution.

Note that when $I$ does not have a linear resolution, the step $i_{3}$ of the minimal graded free resolution of $I$ where nonlinear syzygies first appear is determined, and the results of Eisenbud, Green, Hulek and Popescu mentioned before are recovered. Moreover, we show that the nonlinear syzygies at the $i_{3}$ th step of the resolution are concentrated in degree $i_{3}+3$ and we compute the corresponding Betti number $\beta_{i_{3}, i_{3}+3}$.

If we consider the standard $\mathbb{N}^{n}$-grading on the polynomial ring $R$, monomial ideals are $\mathbb{N}^{n}$-graded modules. In particular, $I$ has a minimal multigraded free resolution as the one in (11) where one substitutes multidegrees $\underline{s} \in \mathbb{N}^{n}$ for degrees $j \in \mathbb{N}$. The number of syzygies of multidegree $\underline{s}$ in the $i$ th syzygy module is denoted by $\beta_{i, \underline{s}}$ and these numbers are called the multigraded Betti numbers of $I$. The multigraded resolution provides a finer numerical information than the graded one. In particular, the graded Betti numbers are recovered from the multigraded ones by $\beta_{i, j}=\sum_{\underline{s} ;|\underline{s}|=j} \beta_{i, \underline{s}}$. In this note, we shall work with the multigraded resolution and we shall prove, in fact, a result which is stronger than the one stated in Theorem 1.1: indeed, the multidegrees $\underline{s}$ such that $\beta_{i_{3}, \underline{s}} \neq 0$ and $|\underline{s}|=i_{3}+3$ are determined, and we shall see that $\beta_{i_{3}, \underline{s}}=1$ for each of these multidegrees.

The minimal graded free resolution of an ideal associated to a graph and, in particular, its Betti numbers depend in general on the characteristic of the field $K$; see [10, Section 4]. Nevertheless, whenever a property of the resolution can be characterized in terms of the combinatorial data of the graph $G(I)$ or any other graph associated to the generating 
monomials of $I$, this will not occur. The aforementioned result of Fröberg illustrates this observation. So does our Theorem 1.1 where the index $i_{3}$ and the corresponding Betti number $\beta_{i_{3}, i_{3}+3}$ are computed independently of the characteristic of $K$. This happens with other Betti numbers as observed by several authors in the case where $I$ is an edge ideal. For example, there is a formula for $\beta_{1,3}$ in [5]; see [13, Proposition 6.6.3]. This result is later recovered in [11] where the first row of the Betti diagram of edge ideals is studied. More precisely, a closed formula for $\beta_{i-2, i}$ is given for $i \leq 5$, and for all $i$ when $G(I)$ has no induced 4-cycles. In [10], it is shown that for all $i \geq 0, \beta_{i, 2(i+1)}$ is the number of induced subgraphs of $G(I)$ consisting of $i+1$ disjoint edges. We shall see in Lemma 4.1 that the same result holds for arbitrary ideals associated to graphs.

In Section 2, we recall Fröberg's characterization of edge ideals having a linear resolution and review some refinements obtained recently. In particular, the results of Eisenbud, Green, Hulek and Popescu cited before are mentioned. In Section 3, we focus on a special class of edge ideals whose resolution is nonlinear. The Betti diagram of these ideals is completely determined in Proposition 3.1 and we can observe that Theorem 1.1 holds in this case. This example plays an important role in the proof of the main result in Section 4 , Theorem 4.6, that computes the number of nonlinear syzygies of smallest degree of ideals associated to graphs. In Section 5, a description of the shape of the Betti diagram is given in Theorem 5.2. This is the last ingredient for the proof of Theorem 1.1 which is given at the very end of the paper.

\section{FrÖBERG'S RESUlt AND ITS LATER REFINEMENTS}

Before stating Fröberg's result, let us recall some definitions.

Definition 2.1. Let $G$ be a graph with vertex set $V$ and edge set $E$. Given $t \geq 3$, a $t$-cycle in $G$ is a subgraph of $G$ whose edges are of the form $\left(v_{1}, v_{2}\right),\left(v_{2}, v_{3}\right), \ldots,\left(v_{t}, v_{1}\right)$ where $v_{1}, \ldots, v_{t}$ are distinct elements in $V$. A $t$-cycle $C$ in $G$ has a chord if $\left(v_{i}, v_{j}\right) \in E$ for some $1 \leq i<j \leq t$ such that $\left(v_{i}, v_{j}\right)$ is not an edge of $C$. An induced (or minimal) $t$-cycle is a $t$-cycle in $G$ with no chord. More generally, a subgraph of $G$ is induced if its edge set contains all the elements in $E$ joining two distinct elements in its vertex set. A graph $G$ is said to be chordal if it has no induced $t$-cycle with $t \geq 4$.

Theorem 2.2 ([6, Theorem 1]). An edge ideal I has a linear resolution if and only if the graph $G(I)^{c}$ is chordal.

This result has recently been recovered by Eisenbud, Green, Hulek and Popescu. Moreover, if the resolution is nonlinear, they also determine the step in the resolution where nonlinear syzygies first appear:

Theorem 2.3 (4, Theorem 2.1]). If I is an edge ideal with nonlinear resolution, the smallest integer $r \geq 4$ such that $\beta_{r-3, j} \neq 0$ for some $j \geq r$ coincides with the smallest integer $r \geq 4$ such that $G(I)^{c}$ has an induced $r$-cycle.

The general monomial case is usually reduced to the squarefree monomial case via polarization but one can sometimes provide a direct result. This is illustrated by the following result that gathers two characterizations that have been obtained independently. Recall that a homogeneous ideal is linearly presented when the module of its first syzygies is generated by linear ones. We will refer to a vertex $v$ such that the loop $(v, v)$ belongs to $E_{G(I)}$ as a square vertex of $G(I)$. The edge graph $G(I)^{*}$ of $G(I)$ featured in Proposition 2.4 (3) has vertex set $V_{G(I)^{*}}:=\{(1), \ldots,(m)\}$ and edge set $E_{G(I)^{*}}:=\left\{(i, j) ; 1 \leq i<j \leq m / \operatorname{lcm}\left(f_{i}, f_{j}\right) \neq 1\right\}$. 
It is always a simple graph. The distance between two vertices of $G(I)^{*}$ is the minimum length of a path connecting them, and the diameter of $G(I)^{*}$ is the longest distance, i.e., the longest shortest path, between any two of its vertices (the diameter of a nonconnected graph is infinite).

Proposition 2.4. Given an ideal I generated by monomials of degree two, the following are equivalent:

(1) I is linearly presented.

(2) The edge ideal $I_{s q}$ obtained from $I$ by removing its square generators is linearly presented, any two square vertices of $G(I)$ are adjacent, and for any edge $\left(v_{i}, v_{j}\right)$ of $G(I)$ that is not a loop and any square vertex $v_{k}, v_{k}$ is adjacent to either $v_{i}$ or $v_{j}$.

(3) The graph $G(I)^{*}$ has diameter $\leq 2$.

(11) $\Leftrightarrow$ (2) is [4, Proposition 2.3 (a)] and (11) $\Leftrightarrow$ (3) is [2, Lemma 4.28]. Observe that using Theorem 2.3 in order to translate the condition on $I_{s q}$ in (2) in terms of the graph $G\left(I_{s q}\right)^{c}=G(I)^{c}$, one can easily check directly that (2) $\Leftrightarrow(3)$.

Finally, when $I$ is linearly presented one has the following result:

Proposition 2.5 ([4, Proposition 2.3 (b)]). If I is an ideal generated by monomials of degree two which is linearly presented, let $I_{s q}$ be the edge ideal obtained from $I$ by removing its square generators. Then, I has a linear resolution if and only if $I_{s q}$ has. Moreover, when this does not occur, the step of the minimal graded free resolution where nonlinear syzygies first appear is the same for $I$ and $I_{s q}$.

Remark 2.6. Theorem 1.1 contains Theorem 2.3 and Propositions 2.4 and 2.5 ,

- In order to recover Theorem 2.3, observe that if $I$ is an edge ideal, $G(I)$ has an induced subgraph consisting of two disjoint edges if and only if $G(I)^{c}$ has an induced 4-cycle. Note that this claim is wrong if we do not assume that $I$ is squarefree.

- It is easy to check that $G(I)$ has no induced subgraph consisting of two disjoint edges if and only if $G(I)^{*}$ has diameter $\leq 2$ and hence, Theorem 1.1 (11) contains Proposition 2.4

- Finally, observe that if we assume that $G(I)$ has no induced subgraph consisting of two disjoint edges, i.e., that $I$ is linearly presented by Theorem 1.1(1), then the other combinatorial conditions in Theorem 1.1 and also the value of $i_{3}$ in Theorem 1.1 (2) only depend on the graph $G(I)^{c}=G\left(I_{s q}\right)^{c}$ and hence, Proposition 2.5 follows.

\section{An eXample Where the Whole Betti diagram is Determined}

We focus in this section on the case where $I$ is the edge ideal generated by all the squarefree monomials of degree two in the variables $x_{1}, \ldots, x_{n}$ except $x_{1} x_{2}, x_{2} x_{3}, \ldots, x_{n} x_{1}$. Then, $G(I)^{c}$ is an $n$-cycle. In [4, Example 2.2], it is shown that the syzygies of $I$ are linear in steps $0, \ldots, n-4$ and that $\beta_{n-3, n}=1$. The whole resolution of $I$ is given by the following result:

Proposition 3.1. Given $n \geq 4$, if $I \subset R:=K\left[x_{1}, \ldots, x_{n}\right]$ is an edge ideal such that $G(I)^{c}$ is a cycle with $n$ vertices, the minimal graded free resolution of $I$ is

$$
0 \longrightarrow R(-n) \longrightarrow R(-n+2)^{\beta_{n-4}} \longrightarrow \cdots \longrightarrow R(-2)^{\beta_{0}} \longrightarrow I \longrightarrow 0
$$

where for all $i, 0 \leq i \leq n-4, \beta_{i}:=n \frac{i+1}{n-i-2}\left(\begin{array}{c}n-2 \\ i+2\end{array}\right)$. 
Remark 3.2. Using the Auslander-Buchsbaum formula, the above result implies that $R / I$ is a Gorenstein ring of dimension two. The well-know symmetry of the Betti numbers when $R / I$ is Gorenstein can be observed checking easily in our formula that $\beta_{n-4-i}=\beta_{i}$ for all $i, 0 \leq i \leq n-4$.

In order to prove Proposition 3.1, we use a special case of Hochster's formula (see [9]) for an edge ideal as stated in [11, Proposition 1.2]: denoting by $V:=\{(1), \ldots,(n)\}$ the vertex set of $G(I)$,

$$
\beta_{i, j}=\sum_{S \subseteq V ;|S|=j} \operatorname{dim}_{K} \tilde{H}_{j-i-2}\left(\Delta\left(G_{S}^{c}\right), K\right), \quad \forall i, j \geq 0,
$$

where $G_{S}$ denotes the induced subgraph of $G(I)$ on the vertex set $S, G_{S}^{c}$ is its complement (in the vertex set $S$ ), and $\Delta\left(G_{S}^{c}\right)$ is the clique complex of $G_{S}^{c}$. Note that $\Delta\left(G_{S}^{c}\right)$ is a subcomplex of $\Delta:=\Delta\left(G(I)^{c}\right)$, the clique complex of $G(I)^{c}$. Indeed, $\Delta\left(G_{S}^{c}\right)$ coincides with $\Delta_{S}$, the subcomplex of $\Delta$ whose vertex set is $S$.

Since $G(I)^{c}$ is a cycle, the only subcomplex of $\Delta$ with nontrivial homology in degree $>0$ is $\Delta$ itself which reduced homology is $K$ and is concentrated in degree 1 . Thus, $\beta_{n-3, n}=1$ and $\beta_{i, j}=0$ for any other $i, j$ such that $j>i+2$.

One can now determine the first row of the Betti diagram using the formulation of (2) given in [11, Proposition 2.1] when $j=i+2$ :

$$
\beta_{i, i+2}=\sum_{S \subseteq V ;|S|=i+2}\left(\# \operatorname{comp}\left(G_{S}^{c}\right)-1\right), \quad \forall i \geq 0 .
$$

Subgraphs with one component have no contribution in (3) so $\beta_{i, i+2}=0$ if $i+2 \geq n-1$. Since the number $k$ of components of $G_{S}^{c}$ satisfies $1 \leq k \leq i+2$ for all $S \subseteq V$ with $|S|=i+2$, one has that for all $i \geq 0$ such that $i+2<n-1$,

$$
\left.\beta_{i, i+2}=\sum_{k=2}^{i+2} \sum_{\substack{S \subseteq V ; \\|S|=i+2 \text { and } \\ \# \operatorname{comp}\left(G_{S}^{c}\right)=k}}(k-1)\right)=\sum_{k=2}^{i+2}(k-1) N(i+2, k)
$$

where $N(i+2, k)$ is the number of induced subgraphs of $G_{S}^{c}$ with $i+2$ vertices and $k$ components. We need the following technical lemma:

Lemma 3.3. Let $i, k, n$ be integers such that $0<k \leq i<n$ and let $C$ be an $n$-cycle. Then, the number of induced subgraphs of $C$ with $i$ vertices and $k$ components is $\frac{n}{k}\left(\begin{array}{c}i-1 \\ k-1\end{array}\right)\left(\begin{array}{c}n-i-1 \\ k-1\end{array}\right)$.

Proof. Let $V:=\{(1), \ldots,(n)\}$ be the vertex set of $C$. Given a subset $S$ of $V$, the induced subgraph $C_{S}$ of $C$ with vertex set $S$ can be represented by a vector $w_{S}$ of length $n$ whose $\ell$ th entry is 1 if $(\ell) \in S$ and 0 otherwise. From now on, we identify the induced subgraph $C_{S}$ of $C$ to the vector $w_{S}$.

This identification can be used to compute the number of induced subgraphs of $C$ with $i$ vertices and $k$ components. Indeed, the number $i$ of vertices in $w_{S}$ is the number of its nonzero entries, and the number $k$ of components of $w_{S}$ can easily be related to the number of blocks of nonzero entries in $w_{S}$. In order to avoid distinguishing cases as when the vector $w_{S}$ starts/ends with $1 / 0$, we make an easy observation. Consider the set $W$ of vectors $w$ of length $n$ with entries 0 and 1, whose first entry is 1 and last entry is 0 , with $i$ nonzero entries and $k$ blocks of nonzero entries (hence $k$ blocks of zero entries). To each $w$ in $W$, 
we can associate $n$ subgraphs of $C$ with $i$ vertices and $k$ components assigning to the first entry of $w$ one of the vertices in $\{(1), \ldots,(n)\}$. Conversely, each induced subgraph $w_{S}$ of $C$ with $i$ vertices and $k$ components always comes from $k$ vectors $w$ in $W$ depending on which of the blocks of nonzero entries of $w_{S}$ is the first block of $w$. This implies that the number of induced subgraphs of $C$ with $i$ vertices and $k$ components is $\frac{n}{k} \times|W|$. It is an easy exercise to show that the number of elements in $W$ is equal to $\left(\begin{array}{c}i-1 \\ k-1\end{array}\right)\left(\begin{array}{c}n-i-1 \\ k-1\end{array}\right)$ and the result follows.

Applying Lemma 3.3 in (4), one gets that, for all $i<n-3$,

$$
\beta_{i, i+2}=\sum_{k=2}^{i+2}(k-1) \frac{n}{k}\left(\begin{array}{c}
i+1 \\
k-1
\end{array}\right)\left(\begin{array}{c}
n-i-3 \\
k-1
\end{array}\right)=n \sum_{k=1}^{i+1} \frac{k}{k+1}\left(\begin{array}{c}
i+1 \\
k
\end{array}\right)\left(\begin{array}{c}
n-i-3 \\
k
\end{array}\right)
$$

and Proposition 3.1 now follows applying the following combinatorial lemma to $m=n-2$ and $a=i+1$ :

Lemma 3.4. For any two integers $m$ and a such that $1 \leq a<m$,

$$
\sum_{k=1}^{a} \frac{k}{k+1}\left(\begin{array}{c}
m-a \\
k
\end{array}\right)\left(\begin{array}{l}
a \\
k
\end{array}\right)=\frac{a}{m-a+1}\left(\begin{array}{c}
m \\
a+1
\end{array}\right) .
$$

Proof. Let $F$ and $g$ be the two polynomials in $\mathbb{Q}[X]$ defined as follows:

$$
F:=(1+X)^{a}=\sum_{k=0}^{a}\left(\begin{array}{l}
a \\
k
\end{array}\right) X^{k} \quad \text { and } \quad g:=(1+X)^{m-a}=\sum_{k=0}^{m-a}\left(\begin{array}{c}
m-a \\
k
\end{array}\right) X^{k} .
$$

Set $f:=F^{\prime}$ and $G:=\int_{0}^{x} g(u) d u$. Then $f=a(1+X)^{a-1}=\sum_{k=1}^{a} k\left(\begin{array}{l}a \\ k\end{array}\right) X^{k-1}=\sum_{k=1}^{a} k\left(\begin{array}{l}a \\ k\end{array}\right) X^{a-k}$ where the last equality follows from the fact that $X^{k-1}$ and $X^{a-k}$ have the same coefficients in the polynomial $\sum_{k=1}^{a} k\left(\begin{array}{l}a \\ k\end{array}\right) X^{k-1}$ because $k\left(\begin{array}{l}a \\ k\end{array}\right)=k \frac{a !}{k !(a-k) !}=a\left(\begin{array}{l}a-1 \\ k-1\end{array}\right)$ and $(a-k+$ 1) $\left(\begin{array}{c}a \\ a-k+1\end{array}\right)=(a-k+1) \frac{a !}{(a-k+1) !(k-1) !}=a\left(\begin{array}{c}a-1 \\ k-1\end{array}\right)$. On the other hand, $G=\frac{(1+X)^{m-a+1}-1}{m-a+1}=$ $\sum_{k=0}^{m-a} \frac{1}{k+1}\left(\begin{array}{c}m-a \\ k\end{array}\right) X^{k+1}$.

Expressing the polynomial $f G$ in two different ways, one gets that

$$
\frac{a\left((1+X)^{m}-(1+X)^{a-1}\right)}{m-a+1}=\left(\sum_{k=1}^{a} k\left(\begin{array}{l}
a \\
k
\end{array}\right) X^{a-k}\right)\left(\sum_{k=0}^{m-a} \frac{1}{k+1}\left(\begin{array}{c}
m-a \\
k
\end{array}\right) X^{k+1}\right)
$$

and the expected formula now follows determining the coefficient of $X^{a+1}$ in both sides of this equality.

\section{Nonlinear Syzygies of SMallest Degree}

In this section, we compute the number of nonlinear syzygies of $I$ of smallest degree when $I$ does not have a linear resolution. Observe that nonlinear syzygies have degree at least 4. The case where $I$ has nonlinear syzygies of degree 4 happens to be special when $I$ has square generators as we shall observe in Remark 4.8, So let us start characterizing when $I$ has nonlinear syzygies of degree 4 and computing the number of such syzygies when this occurs. It is a direct consequence of the following result that has be proved by Katzman in [10, Lemma 2.2] when $I$ is squarefree. 
Lemma 4.1. Let $I$ be an ideal generated by monomials of degree two. For all $i \geq 0, \beta_{i, 2(i+1)}$ is the number of induced subgraphs of $G(I)$ consisting of $i+1$ disjoint edges.

Proof. If $I$ is not squarefree, one can assume without loss of generality that the square generators of $I$ are $x_{1}^{2}, \ldots, x_{p}^{2}$ for some $p \leq n$. The polarization $I^{\prime}$ of $I$ is the edge ideal obtained by substituting, for all $j \in\{1, \ldots, p\}, x_{j} x_{n+j}$ for $x_{j}^{2}$ where $x_{n+1}, \ldots, x_{n+p}$ are $p$ new variables. Note that the graph $G\left(I^{\prime}\right)$ associated to $I^{\prime}$ is obtained from $G(I)$ by substituting each loop $(j, j)$ in $G(I)(1 \leq j \leq p)$ by the whisker $(j, n+j)$, and two disjoint edges of $G\left(I^{\prime}\right)$ form an induced subgraph of $G\left(I^{\prime}\right)$ if and only if the corresponding edges of $G(I)$ form an induced subgraph of $G(I)$. The result now follows applying [10, Lemma 2.2] to the edge ideal $I^{\prime}$ and using the well-known fact that the graded Betti numbers of $I$ (as $K\left[x_{1}, \ldots, x_{n}\right]$-module) and $I^{\prime}$ (as $K\left[x_{1}, \ldots, x_{n}, x_{n+1}, \ldots, x_{n+p}\right]$-module) are the same.

Corollary 4.2. If $I$ is an ideal generated by monomials of degree two, $\beta_{1,4} \neq 0$ if and only if $G(I)$ has at least one induced subgraph consisting of two disjoint edges. When this occurs,

$$
\beta_{1,4}=\#\{\text { induced induced subgraphs of } G(I) \text { consisting of } 2 \text { disjoint edges }\} \text {. }
$$

We can now start to prove Theorem 4.6 that gives the number of nonlinear syzygies of $I$ of smallest degree (except in the already studied case where $I$ is not squarefree and has nonlinear syzygies of degree 4). We shall use essentially Theorem 2.2, Proposition 3.1 and a result by Gasharov, Hibi and Peeva that we recall below. For all $\underline{s}=\left(s_{1}, \ldots, s_{n}\right) \in \mathbb{N}^{n}$, set $\underline{x} \underline{\underline{s}}:=x_{1}^{s_{1}} \cdots x_{n}^{s_{n}} \in R$. Consider a minimal $\mathbb{N}^{n}$-graded free resolution of $I, \mathbf{F}$, and given any monomial $\underline{x} \underline{\underline{s}}$ in $R$, denote by $\mathbf{F}_{\underline{s}}$ the subcomplex of $\mathbf{F}$ that is generated by the $\mathbb{N}^{n}$-homogeneous basis elements of degrees dividing $\underline{x} \underline{\underline{s}}$.

Theorem 4.3 ([7, Theorem 2.1]). For any monomial $\underline{x} \underline{\underline{s}} \in R, \mathbf{F}_{\underline{s}}$ is a minimal $\mathbb{N}^{n}$-graded free resolution of $I_{\underline{s}}$, the monomial ideal generated by $\left\{\bar{f}_{i} ; f_{i}\right.$ divides $\left.\underline{x} \underline{s}\right\}$.

Together with Proposition 3.1, this result implies the following result:

Proposition 4.4. Assume that $G(I)^{c}$ has an induced $r$-cycle with $r \geq 4$, and consider the vertex set $\left\{\left(i_{1}\right), \ldots,\left(i_{r}\right)\right\}$ of this cycle. Let $\underline{s}$ be the element in $\mathbb{N}^{n}$ such that $\underline{x} \underline{s}=x_{i_{1}} \cdots x_{i_{r}}$. Then, the multigraded Betti numbers $\beta_{i, \underline{s}}$ are equal to 0 for all $i<r-3$, and $\beta_{r-3, \underline{s}}$ is equal to 1 . In particular,

$$
\beta_{r-3, r} \geq \#\left\{\text { induced r-cycles in } G(I)^{c}\right\} .
$$

Proof. Applying Theorem 4.3 to $\underline{x} \underline{\underline{s}}=x_{i_{1}} \cdots x_{i_{r}}$, one gets that the subcomplex $\mathbf{F}_{\underline{s}}$ of $\mathbf{F}$ is a minimal $\mathbb{N}^{n}$-graded free resolution of $I_{\underline{s}}$. In particular, the multigraded Betti number $\beta_{i, \underline{s}}$ coincides with the number of generators of multidegree $\underline{s}$ in the $i$ th step of the minimal graded free resolution of $I_{\underline{s}}$ for all $i$. Now observe that $I_{\underline{s}} \subset R$ is minimally generated by all the square free monomials of degree two in $x_{i_{1}}, \ldots, x_{i_{r}}$ except $r$ of them that correspond to the edges in the induced $r$-cycle of $G(I)^{c}$. Since the minimal generators of $I_{\underline{s}}$ only involve the variables $x_{i_{1}}, \ldots, x_{i_{r}}$, the multigraded Betti numbers of $I_{\underline{s}}$ (as $R$-module) are the same as the multigraded Betti numbers of $I_{\underline{s}} \cap K\left[x_{i_{1}}, \ldots, x_{i_{r}}\right]$ (as $K\left[x_{i_{1}}, \ldots, x_{i_{r}}\right]$-module), and the result follows from Proposition 3.1 where the resolution of $I_{\underline{s}} \cap K\left[x_{i_{1}}, \ldots, x_{i_{r}}\right] \subset K\left[x_{i_{1}}, \ldots, x_{i_{r}}\right]$ is described.

Remark 4.5. Since we have described all the Betti numbers in Proposition 3.1, the above argument carries some additional information on the graded Betti numbers of $I$. Indeed, for all $r \geq 4$ such that $G(I)^{c}$ has an induced $r$-cycle, and for all $i$ such that $0 \leq i \leq r-4$, $\beta_{i, i+2} \geq r \frac{i+1}{r-i-2}\left(\begin{array}{c}r-2 \\ i+2\end{array}\right)$. 
Theorem 4.6. Assume that $I$ does not have a linear resolution, and let $r$ be the smallest integer $(\geq 4)$ such that $\beta_{i, r} \neq 0$ for some $i \leq r-3$. If $r=4$, assume moreover that $I$ is squarefree. Then, $r$ is the smallest integer such that $G(I)^{c}$ has an induced $r$-cycle and

$$
\beta_{r-3, r}=\#\left\{\text { induced r-cycles in } G(I)^{c}\right\} \text {. }
$$

Proof. Consider $\underline{s} \in \mathbb{N}^{n}$ with $|\underline{s}|=r$ such that $\beta_{i, \underline{s}}>0$ for some $i \leq r-3$.

We first assume that $\underline{x} \underline{\underline{s}}=x_{i_{1}} \cdots x_{i_{r}}$ with $i_{1}, \ldots, i_{r}$ all different. Then the ideal $I_{s}$ is squarefree. Moreover, it has a nonlinear resolution by Theorem 4.3. Applying Theorem 2.2 to the ideal $J:=I_{\underline{s}} \cap K\left[x_{i_{1}}, \ldots, x_{i_{r}}\right]$, one gets that the complement $G(J)^{c}$ of $G(J)$ (in the vertex set $\left.\left\{\left(i_{1}\right), \ldots,\left(i_{r}\right)\right\}\right)$ has an induced $\ell$-cycle for some $\ell \leq r$. This provides an induced $\ell$-cycle in $G(I)^{c}$. By Proposition 4.4, $\beta_{\ell-3, \ell}>0$ and $\ell \geq r$ by minimality of $r$. Thus $\ell=r$, and we have shown that if $\underline{s} \in \mathbb{N}^{n}$ is such that $\beta_{i, \underline{s}}>0$ for some $i \leq r-3$ and $\underline{x}^{\underline{s}}=$ $x_{i_{1}} \cdots x_{i_{r}}$ is squarefree, then $G(I)^{c}$ has an induced $r$-cycle with vertex set $\left\{\left(i_{1}\right), \ldots,\left(i_{r}\right)\right\}$. By Proposition 4.4, this implies that $\beta_{i, \underline{s}}=0$ for all $i<r-3$ and $\beta_{r-3, \underline{s}}=1$.

Assume now that the monomial $\underline{x} \underline{s}$ is not squarefree and let us see that this leads to a contradiction. If $I_{\underline{s}}$ was squarefree, one could apply the same argument as before and find an induced $\ell$-cycle in $G(I)^{c}$ with $\ell \geq r$ by minimality of $r$. On the other hand, this cycle should be supported on vertices corresponding to variables dividing the monomial $\underline{x} \underline{\underline{s}}$ which is not squarefree and has degree $r$, and hence $\ell<r$. Thus, $I_{\underline{s}}$ is not squarefree. In particular, $I$ is not squarefree and hence $r \geq 5$ by hypothesis. Consider the ideal $J:=I_{\underline{s}} \cap K\left[x_{i_{1}}, \ldots, x_{i_{q}}\right]$ where $\left\{i_{1}, \ldots, i_{q}\right\}$ is the support of the monomial $\underline{x} \underline{\underline{s}}$, and assume without loss of generality that the variables $x_{i}$ with $i \in\left\{i_{1}, \ldots, i_{q}\right\}$ such that $x_{i}^{2}$ is a generator of $J$ are $x_{i_{1}}, \ldots, x_{i_{p}}(p \leq q)$. The polarization $J^{\prime}$ of $J$ is the edge ideal obtained by substituting, for all $j \in\{1, \ldots, p\}, x_{i_{j}} x_{n+j}$ for $x_{i_{j}}^{2}$ where $x_{n+1}, \ldots, x_{n+p}$ are $p$ new variables. Since the graded Betti numbers of $J$ (as $K\left[x_{i_{1}}, \ldots, x_{i_{q}}\right]$-module) and $J^{\prime}$ (as $K\left[x_{i_{1}}, \ldots, x_{i_{q}}, x_{n+1}, \ldots, x_{n+p}\right]$-module) are the same, by applying Theorem 2.2 to the ideal $J^{\prime}$, one gets that the complement $G\left(J^{\prime}\right)^{c}$ of the simple graph $G\left(J^{\prime}\right)$ has an induced $\ell$-cycle for some $\ell \leq p+q$. Denote this $\ell$-cycle by $C$. Note that the simple graph $G\left(J^{\prime}\right)$ associated to $J^{\prime}$ is obtained from $G(J)$ by substituting each loop $\left(i_{j}, i_{j}\right)(1 \leq j \leq p)$ by the whisker $\left(i_{j}, n+j\right)$. We shall show in Lemma 4.7 below that no induced $t$-cycle of $G\left(J^{\prime}\right)^{c}$ with $t \geq 5$ passes through any of the vertices $(n+1), \ldots,(n+p)$. This implies that if $\ell \geq r \geq 5$, then the vertex set of $C$ should be contained in $\left\{\left(i_{1}\right), \ldots,\left(i_{q}\right)\right\}$, and hence $\ell \leq q<r$, a contradiction. Thus $\ell$ has to be strictly smaller than $r$ but, by Proposition 4.4, this implies that $\beta_{\ell-3, \ell}>0$ and by minimality of $r$, one also gets a contradiction.

We have shown that if $\underline{s} \in \mathbb{N}^{n}$ is such that $|\underline{s}|=r$ and $\beta_{i, \underline{s}}>0$ for some $i \leq r-3$, then the monomial $\underline{\underline{s}}=x_{i_{1}} \cdots x_{i_{r}}$ is squarefree and $G(I)^{c}$ has an induced $r$-cycle with vertex set $\left\{\left(i_{1}\right), \ldots,\left(i_{r}\right)\right\}$. Moreover, $\beta_{i, \underline{s}}=0$ for all $i<r-3$ and $\beta_{r-3, \underline{s}}=1$, and the result follows using Proposition 4.4 .

Lemma 4.7. Let $G$ be a simple graph and $v$ a vertex of $G$ of order 1. Then, no induced $t$-cycle of the complement $G^{c}$ of $G$ has $v$ in its vertex set if $t \geq 5$.

Proof. Let $v^{\prime}$ be the vertex of $G$ adjacent to $v$. Consider $S$, a subset with $t$ elements of the vertex set of $G$ containing $v$. The order of the vertex $v$ in the induced subgraph $G_{S}^{c}$ of $G^{c}$ with vertex set $S$ is either $t-2$ if $v^{\prime} \in S$, or $t-1$ otherwise. Since $t-1$ and $t-2$ are strictly bigger that 2 if $t \geq 5$ and the order of any vertex in a cycle is 2 , the result follows.

Remark 4.8. If $I$ is an edge ideal and $r=4$, one can easily check that the value of $\beta_{1,4}$ obtained in Theorem 4.6 coincides with the one in Corollary 4.2, Nevertheless, when $I$ is 
not squarefree and $r=4$, the correct value of $\beta_{1,4}$ is the one given in Corollary 4.2 and the formula in Theorem 4.6 may be wrong as the following example shows: if we consider the ideal $I=\left(x_{1}^{2}, x_{1} x_{3}, x_{3} x_{5}, x_{5} x_{2}, x_{2} x_{4}, x_{4} x_{1}\right) \subset \mathbb{Q}\left[x_{1}, \ldots, x_{5}\right]$, the complement $G(I)^{c}$ of $G(I)$ has no induced 4-cycles while $\beta_{1,4}=1$. The Betti diagram can be obtained using [1] or any other program devoted to computations in algebraic geometry and commutative algebra like Singular, CoCoA or Macaulay2. The unique induced subgraph of $G(I)$ that makes a contribution to $\beta_{1,4}$ in the value given by Lemma 4.1 is the one formed by the loop and the horizontal edge in its representation below.

\begin{tabular}{|l|llll|}
\hline & 0 & 1 & 2 & 3 \\
\hline 2 & 6 & 7 & 1 & - \\
3 & - & 1 & 3 & 1 \\
\hline
\end{tabular}

The Betti diagram of $I$.

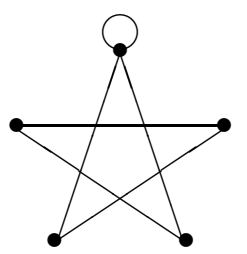

The graph $G(I)$ associated to $I$.

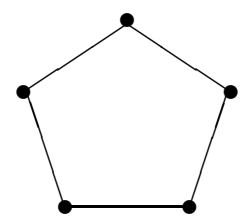

The complement $G(I)^{c}$ of $G(I)$.

Remark 4.9. The proof of Theorem 4.6 carries some additional information: a multidegree $\underline{s} \in \mathbb{N}^{n}$ contributes to $\beta_{r-3, r}$ if and only if $\underline{x} \underline{s}$ is the product of the vertices involved in an induced $r$-cycle of $G(I)^{c}$. Moreover, for such an $\underline{s}$, the corresponding multigraded Betti number $\beta_{r-3, \underline{s}}$ is equal to 1 .

\section{The shape of the Betti diagram}

The only nonzero entry on the first column of the Betti diagram of $I$ is $\beta_{0,2}=\# E_{G(I)}$. The following result of Katzman provides some information on the shape of the Betti diagram when $I$ is an edge ideal.

Lemma 5.1 ([10, Lemma 2.2]). For any edge ideal, $\beta_{i, j}=0$ for all $i \geq 0$ and $j>2(i+1)$.

In terms of the Betti diagram, this means that the entries below the diagonal through $\beta_{0,2}$ are zero. Our next result improves this statement.

Theorem 5.2. Consider an ideal $I \subset K\left[x_{1}, \ldots, x_{n}\right]$ generated by monomials of degree two, with $K$ an arbitrary field, and let $m$ be its Castelnuovo-Mumford regularity. For all $d \geq 3$, set $i_{d}:=\min _{1 \leq i \leq p}\left\{i ; \beta_{i, i+d} \neq 0\right\}$ if $\beta_{i, i+d} \neq 0$ for some $i \geq 1, i_{d}:=0$ otherwise. If $I$ does not have a linear resolution, then

$$
1 \leq i_{3}<\cdots<i_{m} \leq p
$$

Now we know that the shape of the Betti diagram of an ideal associated to a graph is:

\begin{tabular}{|c|ccccccccccccc|}
\hline & 0 & 1 & $\ldots$ & $i_{3}-1$ & $i_{3}$ & $\ldots$ & $i_{4}-1$ & $i_{4}$ & $\ldots$ & $i_{m}-1$ & $i_{m}$ & $\ldots$ & $p$ \\
\hline 2 & $\bullet$ & $*$ & $\ldots$ & $*$ & $*$ & $\ldots$ & $*$ & $*$ & $\ldots$ & $*$ & $*$ & $\ldots$ & $*$ \\
3 & - & - & $\ldots$ & - & $\bullet$ & $\ldots$ & $*$ & $*$ & $\ldots$ & $*$ & $*$ & $\ldots$ & $*$ \\
4 & - & - & $\ldots$ & - & - & $\ldots$ & - & $\bullet$ & $\ldots$ & $*$ & $*$ & $\ldots$ & $*$ \\
$\vdots$ & & & & & & & & & & & & & \\
$m$ & - & - & $\ldots$ & - & - & $\ldots$ & - & - & $\ldots$ & - & $\bullet$ & $\ldots$ & $*$ \\
\hline
\end{tabular}

$\bullet=$ nonzero entry $;-=$ zero entry $; *=$ entry that may be zero or not.

This result will be a direct consequence of our last lemma. Recall that given a simplicial complex $\Delta$ and a vertex $x$ of $\Delta$, the subcomplex link $(x, \Delta)$ of $\Delta$ is defined by

$$
\operatorname{link}(x, \Delta):=\{F \in \Delta ; x \notin F \text { and } F \cup\{x\} \in \Delta\} \text {. }
$$


Lemma 5.3. Assume that $I$ is an edge ideal with nonlinear resolution. Using the notations in Theorem 5.2, if $i_{d} \geq 1$ for some $d \geq 3$, then there exists $i<i_{d}$ such that $\beta_{i, i+d-1} \neq 0$.

Proof. Let $V$ be the vertex set of the graph $G(I)$, and consider $d, 3 \leq d \leq m$, such that $i_{d} \geq 1$. Then, $\beta_{i_{d}, i_{d}+d} \neq 0$ and $\beta_{i, i+d}=0$ for all $i, 0 \leq i<i_{d}$. Using Hochster's formula as stated in (2), we have that there exists $S \subseteq V$ with $|S|=i_{d}+d$ such that $\widetilde{H}_{d-2}\left(\Delta\left(G_{S}^{c}\right), K\right) \neq 0$ while $\widetilde{H}_{d-2}\left(\Delta\left(G_{S^{\prime}}^{c}\right), K\right)=0$ for any subset $S^{\prime} \subset V$ with $\left|S^{\prime}\right|<|S|$.

For $x \in S$, denote by $S_{x}$ the vertex set of $\operatorname{link}\left(x, \Delta\left(G_{S}^{c}\right)\right)$. We claim that there exists a vertex $x \in S$ such that $\left|S_{x}\right|<|S \backslash\{x\}|$. Otherwise, every pair of vertices in $S$ would be linked in $\Delta\left(G_{S}^{c}\right)$ and hence $\Delta\left(G_{S}^{c}\right)$ would be an $\left(i_{d}+d\right)$-simplex. Thus, $\widetilde{H}_{j}\left(\Delta\left(G_{S}^{c}\right), K\right)=0$ for all $j \neq i_{d}+d-2>d-2$, a contradiction with the fact that $\widetilde{H}_{d-2}\left(\Delta\left(G_{S}^{c}\right), K\right) \neq 0$.

So we can choose a vertex $x \in S$ such that $\left|S_{x}\right|<|S \backslash\{x\}|$ and consider the following long exact sequence introduced in [8],

$$
\cdots \rightarrow \widetilde{H}_{d-3}\left(\operatorname{link}\left(x, \Delta\left(G_{S}^{c}\right), K\right) \rightarrow \widetilde{H}_{d-2}\left(\Delta\left(G_{S}^{c}\right), K\right) \rightarrow \widetilde{H}_{d-2}\left(\Delta\left(G_{S}^{c}\right)_{S \backslash\{x\}}, K\right) \rightarrow \cdots\right.
$$

where $\Delta\left(G_{S}^{c}\right)_{S \backslash\{x\}}$ is the subcomplex of $\Delta\left(G_{S}^{c}\right)$ whose vertex set is $S \backslash\{x\}$. Note that $\Delta\left(G_{S}^{c}\right)_{S \backslash\{x\}}=\Delta\left(G_{S \backslash\{x\}}^{c}\right)$ and so $\widetilde{H}_{d-2}\left(\Delta\left(G_{S}^{c}\right)_{S \backslash\{x\}}, K\right)=0$ because $|S \backslash\{x\}|<|S|$. Since $\widetilde{H}_{d-2}\left(\Delta\left(G_{S}^{c}\right), K\right) \neq 0$, one gets that $\widetilde{H}_{d-3}\left(\operatorname{link}\left(x, \Delta\left(G_{S}^{c}\right)\right), K\right) \neq 0$.

Since we have chosen $x \in S$ such that $\left|S_{x}\right|<|S \backslash\{x\}|=i_{d}+d-1$ and observing that $\operatorname{link}\left(x, \Delta\left(G_{S}^{c}\right)\right)=\Delta\left(G_{S_{x}}^{c}\right)$, we get that $\widetilde{H}_{d-3}\left(\Delta\left(G_{S_{x}}^{c}\right), K\right) \neq 0$ with $j:=\left|S_{x}\right| \leq i_{d}+d-2$. Using again Hochster's formula, we deduce that $\beta_{j-d+1, j}>0$. Setting $i:=j-d+1 \leq$ $i_{d}+d-2-d+1=i_{d}-1<i_{d}$, one has that $\beta_{i, i+d-1}>0$ and we are done.

Proof of Theorem 5.2. The result trivially holds if $m=3$ and since a monomial ideal and its polarization have the same Betti diagram, let us assume that $m \geq 4$ and that $I$ is an edge ideal. Starting with $d=m$ and applying Lemma 5.3. one gets that there is a nonzero entry on the $(d-1)$ th row, i.e., $i_{d-1} \geq 1$, and that $i_{d-1}<i_{d}$. Iterating the argument until $d=4$, the result follows.

Remark 5.4. (1) As observed in [10, Section 4], the Betti diagram (in particular the number of rows and columns) may depend on the characteristic of the field $K$. Nevertheless, it will have the shape described in Theorem 5.2 in any characteristic.

(2) By Theorem 1.1, $i_{3}$ and $\beta_{i_{3}, i_{3}+3}$ do not depend on the characteristic of $K$. The four graphs given in [10, Appendix A] show that the characteristic of $K$ may be relevant for the computation of $\beta_{i_{4}, i_{4}+4}$.

All the ingredients are now available to prove the result stated in the introduction:

Proof of Theorem 1.1. (11): it is Corollary 4.2.

(2): Assume that $i_{3}>1$. Then $G(I)$ has no induced subgraph consisting of two disjoint edges by (11), Theorem 4.6 implies that $G(I)^{c}$ has an induced cycle of length $\geq 5$ and gives the values of $i_{3}$ and $\beta_{i_{3}, i_{3}+3}$, and the last statement is a consequence of Theorem 5.2 . Conversely, if $G(I)$ has no induced subgraph consisting of two disjoint edges then $i_{3} \neq 1$ by (11), and if $G(I)^{c}$ has an induced cycle of length $\geq 5$ then $i_{3} \neq 0$ by Proposition 4.4.

(3): The 'if and only if' follows from (11) and (2), and the last statement is a consequence of Theorem 5.2 . 


\section{ACKnowledgements}

Research partially supported by MTM2007-61444, Ministerio de Educación y Ciencia (Spain). The authors thank Isabel Bermejo and Aron Simis for helpful conversations.

\section{REFERENCES}

[1] D. Bayer and M. Stillman, Macaulay, a system for computation in algebraic geometry and commutative algebra, available at math.harvard.edu, 1992.

[2] I. Bermejo, P. Gimenez and A. Simis, Polar syzygies in characteristic zero: the monomial case, J. Pure Appl. Algebra 213: 1-21, 2009.

[3] D. Eisenbud, The geometry of syzygies. Graduate Texts in Mathematics 229, Springer, 2005.

[4] D. Eisenbud, M. Green, K. Hulek and S. Popescu, Restricting linear syzygies: algebra and geometry. Compos. Math. 141: 1460-1478, 2005.

[5] S. Eliahou and R. H. Villarreal, The second Betti number of an edge ideal. In: XXXI National Congress of the Mexican Mathematical Society (Hermosillo, 1998), Aportaciones Mat. Comun. 25: 115-119, 1999

[6] R. Fröberg, On Stanley-Reisner rings. In: Topics in Algebra, Part 2 (Warsaw, 1988), Banach Center Publ. 26: 57-70, 1990.

[7] V. Gasharov, T. Hibi and I. Peeva, Resolutions of a-stable ideals. J. Algebra 254: 375-394, 2002.

[8] T. Hibi, Buchsbaum complexes with linear resolutions. J. Algebra 179: 127-136, 1996.

[9] M. Hochster, Cohen-Macaulay rings, combinatorics, and simplicial complexes. In: Ring Theory II (Proc. Second Conf., Univ. Oklahoma, 1975), Lecture Notes in Pure and Appl. Math. 26, Marcel Dekker, 1977.

[10] M. Katzman, Characteristic-independence of Betti numbers of graph ideals. J. Combin. Theory Ser. A 113:435-454, 2006.

[11] M. Roth and A. Van Tuyl, On the linear strand of an edge ideal. Comm. Algebra 35: 821-832, 2007.

[12] A. Simis, W. V. Vasconcelos and R. Villarreal, The integral closure of subrings associated to graphs. $J$. Algebra 199: 281-289, 1998.

[13] R. H. Villarreal, Monomial Algebras. Monographs and Textbooks in Pure and Applied Mathematics 238, Marcel Dekker, 2001.

E-mail address: oscarf@agt.uva.es

E-mail address: pgimenez@agt.uva.es

Depto. de Algebra, Geometría y Topología, Facultad de Ciencias, Universidad de ValLADOLID, 47005 VAlladOLID, SPAiN 